\title{
OPTIMAL DRIFT ON $[0,1]$
}

\author{
SUSAN LEE
}

\begin{abstract}
Consider one-dimensional diffusions on the interval $[0,1]$ of the form $d X_{t}=d B_{t}+b\left(X_{t}\right) d t$, with 0 a reflecting boundary, $b(x) \geq 0$, and $\int_{0}^{1} b(x) d x=1$. In this paper, we show that there is a unique drift which minimizes the expected time for $X_{t}$ to hit 1 , starting from $X_{0}=0$. In the deterministic case $d X_{t}=b\left(X_{t}\right) d t$, the optimal drift is the function which is identically equal to 1 . By contrast, if $d X_{t}=d B_{t}+b\left(X_{t}\right) d t$, then the optimal drift is the step function which is 2 on the interval [1/4,3/4] and is 0 otherwise. We also solve this problem for arbitrary starting point $X_{0}=x_{0}$ and find that the unique optimal drift depends on the starting point, $x_{0}$, in a curious manner.
\end{abstract}

\section{INTRODUCTION AND SUMMARY OF RESULTS}

Let $B_{t}$ be a one-dimensional Brownian motion, and consider the stochastic differential equation $d X_{t}=d B_{t}+b\left(X_{t}\right) d t$ on $[0, \infty)$ with 0 a reflecting boundary. Let $T_{1}=\inf \left\{t>0: X_{t}=1\right\}$ be the first time $X_{t}$ hits 1 , and let $E_{x} T_{1}$ be the expected value of $T_{1}$ starting from $X_{0}=x$. The main question considered in this paper is:

$$
\begin{aligned}
& \text { for each fixed } x_{0}, \text { what drift minimizes } \Phi\left(x_{0}, b\right)=E_{x_{0}} T_{1} \\
& \text { over all } b \text { such that } b \geq 0 \text { and } \int_{0}^{1} b(z) d z=1 \text { ? }
\end{aligned}
$$

The original motivation for this problem was a conjecture proposed by Itai Benjamini and Yuval Peres. Fix $N$, a positive integer. Among all rooted trees with $2^{N}$ leaves at level $N$ such that the root has at least two children, what tree minimizes the expected time for a simple random walk, starting from the root, to reach level $N$ ? Benjamini and Peres conjecture that the (full) binary tree minimizes this expected value.

In the special case of spherically symmetric trees, this conjecture can be verified through standard Markov chain techniques. Recently, Lyons, Pemantle, and Peres [3] showed that for any positive integer $m$, the speed of random walk on a nondegenerate Galton-Watson tree with mean $m$ is strictly smaller than

Received by the editors January 5, 1994.

1991 Mathematics Subject Classification. Primary 60H10; Secondary 60J60, $60 \mathrm{~J} 65$.

Key words and phrases. Stochastic differential equations, diffusion processes, drift, hitting time, optimization.

Research partially supported by the Army Research Office through the Mathematical Sciences Institute at Cornell University. 
the speed of random walk on a deterministic $m$-ary tree. We will show in this paper that contrary to what might be expected by analogy, in the related case of Brownian motion with fixed total drift, the optimal drift is not identically equal to a constant.

Our first result shows that if we relax the positivity constraint on the drift $b$, then there is no optimal drift. Let $\mathscr{A}$ be the class of functions $b$ such that $b \in L^{1}[0,1]$ and $\int_{0}^{1} b(x) d x=1$.

Theorem 1. The infimum of $\Phi\left(x_{0}, b\right)$ over all $b \in \mathscr{A}$ equals 0 .

Let $\gamma_{n}=n e^{n}, \alpha_{n}=e^{-n}$, and $\delta_{n}=\left(1-\alpha_{n}\right) /(n+2)$. We will prove that $\Phi\left(0, b_{n}\right) \rightarrow 0$ as $n \rightarrow \infty$, where $\left\{b_{n}\right\}$ is a sequence of step functions such that

$$
b_{n}(x)= \begin{cases}-\gamma_{n} / 2 & \text { if } 0 \leq x \leq \alpha_{n}, \\ 1 /\left(2 \delta_{n}\right) & \text { if } \alpha_{n}<x \leq 1 .\end{cases}
$$

Because of Theorem 1, it is natural to consider only nonnegative functions, $b \geq 0$. In order to pursue a minimum within a sensible context, we begin with the observation that, under certain restrictions on $b, u(x)=E_{x} T_{1}$ solves the differential equation

$$
\left\{\begin{array}{l}
-1=\frac{1}{2} u^{\prime \prime}+b u^{\prime} \\
u^{\prime}(0)=u(1)=0
\end{array}\right.
$$

This differential equation can be solved explicitly and

$$
u(x)=2 \int_{x}^{1} \int_{0}^{y} e^{-2 \int_{s}^{y} b(z) d z} d s d y=2 \int_{x}^{1} \varphi^{\prime}(y) \int_{0}^{y} \frac{d s}{\varphi^{\prime}(s)} d y,
$$

where $\varphi$ is the natural scale or, more specifically,

$$
\varphi^{\prime}(y)=\exp \left(-2 \int_{0}^{y} b(z) d z\right) .
$$

The expression for $\varphi^{\prime}$ makes sense if we replace $\int_{0}^{y} b(z) d z$ by $b[0, y]$ where $b$ is a probability measure on $[0,1]$ and $b[0, y]$ is the weight that $b$ gives to the interval $[0, y]$. Thus, for each fixed $x_{0}$, we can view $\Phi\left(x_{0}, b\right)=u\left(x_{0}\right)$ as a functional on the space of probability measures on $[0,1]$. (Later, we will construct for each probability measure $b$ a diffusion with drift $b$ and show that $E_{x} T_{1}$ equals $u(x)$.)

Now we will demonstrate that $\Phi$ achieves its minimum in the space of probability measures. Let $\mathscr{A}_{m}$ denote the class of probability measures on $[0,1]$. Fix the starting point $x_{0}$. If $\left\{b_{n}\right\}$ is a sequence of measures in $\mathscr{A}_{m}$ such that $b_{n}$ converges weakly to $b$ as $n \rightarrow \infty$, then $b_{n}[0, y] \rightarrow b[0, y]$ for almost all $y \in[0,1]$. In addition, $e^{-2} \leq \varphi^{\prime} \leq 1$. Thus, by Lebesgue's Dominated Convergence Theorem, $\Phi\left(x_{0}, b_{n}\right) \rightarrow \Phi\left(x_{0}, b\right)$ as $n \rightarrow \infty$. In other words, $\Phi\left(x_{0}, \cdot\right)$ is continuous in the weak ${ }^{*}$ topology. Since $\mathscr{A}_{m}$ is compact under the weak topology, $\Phi\left(x_{0}, \cdot\right)$ must achieve its minimum in $\mathscr{A}_{m}$.

Consider the deterministic equation $d X_{t}=b\left(X_{t}\right) d t$, with the initial condition $X_{0}=x_{0}$. An application of Jensen's inequality shows that, among positive functions $b$ with $\int_{0}^{1} b(z) d z=1$, the function which minimizes the time for $X_{t}$ to hit 1 is the function which is constant on $\left[x_{0}, 1\right]$. By contrast, if $d X_{t}=d B_{t}+b\left(X_{t}\right) d t$, then the optimal drift is not the function which is constant on $\left[x_{0}, 1\right]$. Theorem 2 identifies the unique optimal drift for each starting point $x_{0}$. 
Theorem 2. For each fixed $x_{0}, 0 \leq x_{0}<1$, there is a unique measure $b_{0}$ which achieves the minimum of $\Phi\left(x_{0}, \cdot\right)$ in the space of probability measures on $[0,1]$. This optimal measure $b_{0}$ depends on $x_{0}$ and can be described as follows.

If $0 \leq x_{0} \leq 1 / 4$, then $b_{0}$ has a density which is given by

$$
b_{0}(x)= \begin{cases}2 & \text { if } 1 / 4 \leq x \leq 3 / 4 \\ 0 & \text { otherwise }\end{cases}
$$

If $1 / 4<x_{0}<1 /\left(e^{-2}+1\right)$, then $b_{0}$ is the sum of two measures, a measure concentrated at $x_{0}$ of mass $w>0$ and an absolutely continuous measure with density:

$$
b_{0}(x)= \begin{cases}(1-w) /\left(x_{1}-x_{0}\right) & \text { if } x_{0} \leq x \leq x_{1}, \\ 0 & \text { otherwise. }\end{cases}
$$

The variables, $x_{1}$ and $w$, satisfy

$$
\frac{1}{2}\left(\frac{x_{1}-x_{0}}{1-w}\right)=x_{0} e^{-2 w}=1-x_{1} .
$$

If $x_{0} \geq 1 /\left(e^{-2}+1\right)$, then $b_{0}$ is the unit mass concentrated at $x_{0}$.

The value of $\Phi\left(x_{0}, b_{0}\right)$ is as follows:

$$
\Phi\left(x_{0}, b_{0}\right)= \begin{cases}\frac{1}{2}-x_{0}^{2} & \text { if } 0 \leq x_{0} \leq 1 / 4, \\ \left(1-x_{1}\right)\left[3-2 x_{0}-x_{1}\right] & \text { if } 1 / 4<x_{0}<1 /\left(e^{-2}+1\right), \\ \left(1-x_{0}\right)\left[1+x_{0}\left(2 e^{-2}-1\right)\right] & \text { if } 1 /\left(e^{-2}+1\right) \leq x_{0}<1\end{cases}
$$

It is difficult to explain this answer intuitively. We began our investigation with the case $x_{0}=0$ and discovered that, among all step functions $b$ with $\int_{0}^{1} b(x) d x=1$ that are constant on $[c, 1-c]$ and 0 otherwise, $\Phi(0, b)$ achieves its minimum at $c=1 / 4$. In particular, the value of $\Phi$ at $c=1 / 4$ is strictly less than the value of $\Phi$ at $c=0$. Later, we observed that if $b_{r}$ is the reflection of $b$ about $1 / 2$, then $\Phi\left(0, b_{r}\right)=\Phi(0, b)$. Hence, in the presence of strict convexity, the minimum must be symmetric. In hindsight, it is not surprising that the drift is turned off near 0 , where reflection occurs, and near 1 , when we have almost reached our goal. (Of course, by symmetry one of these reasons is redundant.)

We believed and then proved that the step function defined in (3) is the right answer mainly because a variational calculation showed the following. Let $G(x, y)$ be the Green's function for our process killed when it hits 1 , and let $u(x)=E_{x} T_{1}$. If $b$ is minimal, the the product $G(x, x) u^{\prime}(x)$ must be constant on the set $\{x: b(x)>0\}$. Further calculations showed that if $G(x, x) u^{\prime}(x)$ is constant, then $b^{\prime}(x)=0$. Therefore, $b$ must be constant where it is nonzero. Similar calculations allowed us to determine the optimal drift for an arbitrary starting point.

Theorem 2 addresses the problem of minimizing the expected time to hit 1 . It is also interesting to let $T_{0}=\inf \left\{t>0: X_{t}=0\right\}$ be the first time $X_{t}$ hits 0 and ask: what drift will maximize $P_{x}\left(T_{1}<T_{0}\right)$, the probability that $X_{t}$ hits 1 before it hits 0 , starting from $X_{0}=x$ ? This time the answer is very simple. 
Theorem 3. For each fixed $x$, the unique measure which achieves the maximum of $\Psi(x, b)=P_{x}\left(T_{1}<T_{0}\right)$ in $\mathscr{A}_{m}$ is the unit mass concentrated at $x$.

Note that if the starting position $X_{0}=x$ is close to 1 , then the unit mass concentrated at $x$ both maximizes the probability of hitting 1 before hitting 0 and minimizes the expected time to reach 1 . We have no probabilistic explanation for this phenomenon yet.

Furthermore, if $b$ is the measure concentrated at 0 of mass $w$, then the diffusion $d X_{t}=d B_{t}+b\left(X_{t}\right) d t$ has a simple interpretation. It is skew Brownian motion. In other words, given a reflecting Brownian motion on $[0, \infty)$, change the sign of the excursions away from 0 independently, so that a given excursion is positive with probability $\alpha=1 /\left(e^{-2 w}+1\right)$ and is negative with probability $1-\alpha$. The resulting diffusion is $X_{t}$. See Harrison and Shepp [2], and Engelbert and Schmidt [1] for more details.

Lastly, we will show that in some sense, there exists a diffusion with drift $b$ where $b$ is a measure. Given a measure $b$, let $\varphi^{\prime}$ be the right-continuous function

$$
\varphi^{\prime}(b, z)= \begin{cases}e^{-2 b[0, z]} & \text { if } z \geq 0 \\ e^{2 b(z, 0)} & \text { if } z<0 .\end{cases}
$$

Let $\varphi(b, x)$ be a function such that $\varphi(b, x)-\varphi\left(b, x_{0}\right)=\int_{x_{0}}^{x} \varphi^{\prime}(b, z) d z$. Call $\varphi$ the natural scale.

Definition. A diffusion process, $X_{t}$, will be called a solution to the stochastic differential equation (SDE) $d X_{t}=d B_{t}+b\left(X_{t}\right) d t$ if there exists a natural scale, $\varphi(b, x)$, such that $\varphi\left(b, X_{t}\right)$ is a martingale and $\langle X\rangle_{t}=t$.

In order to take into account reflection at 0 , we observe that if $b$ is a measure on $[0,1]$ with density $b(x)$ and $d X_{t}=d B_{t}+b\left(X_{t}\right) d t$, then the expected time for $X_{t}$ to hit 1 , with 0 a reflecting boundary, starting from $X_{0}=x$, equals the expected time for $X_{t}$ to exit $(-1,1)$, starting from $X_{0}=x$, where $b(x)$ is extended to be an odd function about 0 . Analogously, we will define what it means for a measure $\bar{b}$ to be the odd reflection of a measure $b$ and then prove:

Theorem 4. There exists a solution to $d X_{t}=d B_{t}+\bar{b}\left(X_{t}\right) d t$. Moreover, the expected time for $X_{t}$ to exit $(-1,1)$, starting from $X_{0}=x$, equals $u(x)$ where $u$ is the function defined in (2).

The interested reader can find in Engelbert and Schmidt [1] necessary and sufficient conditions for the existence and uniqueness of a solution to a SDE with drift where the drift is a measure.

The organization of the paper is as follows. In $\S 2$, we will prove Theorem 1 and show that the infimum is not achieved in $L^{1}[0,1]$. The core of the paper is $\S 3$, where we will prove Theorem 2 , namely, that in $\mathscr{A}_{m}$, the infimum of $\Phi\left(x_{0}, \cdot\right)$ is achieved by the measure $b_{0}$ specified in Theorem 2 . Then we will prove Theorem 3, i.e., we will prove that if the starting point is $x$, then the unit mass concentrated at $x$ maximizes the probability of hitting 1 before hitting 0 . Finally, in $\S 4$, we will prove Theorem 4.

\section{Proof of Theorem 1 -Infimum is Not AChieved in $L^{1}$}

Let $\mathscr{A}$ be the class of real-valued functions $b$ such that $b \in L^{1}[0,1]$ and $\int_{0}^{1} b(x) d x=1$. Let $\varphi^{\prime}(b, x)=\exp \left(-2 \int_{0}^{x} b(z) d z\right)=\exp (-2 b[0, x])$, and let 
$\Phi\left(x_{0}, b\right)$ be the functional

$$
\Phi\left(x_{0}, b\right)=2 \int_{x_{0}}^{1} \int_{0}^{x} e^{-2 b(s, x]} d s d x=2 \int_{x_{0}}^{1} \varphi^{\prime}(b, x) \int_{0}^{x} \frac{d s}{\varphi^{\prime}(b, s)} d x .
$$

Let $\left\{b_{n}\right\}$ be the sequence of functions defined in (1). We will show that $\Phi\left(0, b_{n}\right) \rightarrow 0$ as $n \rightarrow \infty$. (Of course, there is no $b$ in $\mathscr{A}$ such that $\Phi(0, b)$ equals 0.) For ease of notation, we will write $\Phi\left(b_{n}\right)$ instead of $\Phi\left(0, b_{n}\right)$. We will also write $\varphi_{n}^{\prime}(x)$ instead of $\varphi^{\prime}\left(b_{n}, x\right)$. Each function $b_{n}$ is in $\mathscr{A}$ because $\left(1-\alpha_{n}\right) / 2 \delta_{n}-\alpha_{n} \gamma_{n} / 2=1$. In the calculations below, we will repeatedly use without comment the equality $\gamma_{n} \alpha_{n}=n$. By the definition of natural scale,

$$
\varphi_{n}^{\prime}(x)=e^{-2 b_{n}[0, x]}= \begin{cases}e^{\gamma_{n} x} & \text { if } 0 \leq x \leq \alpha_{n}, \\ e^{n} e^{-\left(x-\alpha_{n}\right) / \delta_{n}} & \text { if } \alpha_{n}<x \leq 1 .\end{cases}
$$

Let $M_{n}(x)=\int_{0}^{x} e^{2 b_{n}[0, s]} d s$. If $0 \leq x \leq \alpha_{n}$, then

$$
M_{n}(x)=\int_{0}^{x} e^{-\gamma_{n} s} d s=-\frac{1}{\gamma_{n}}\left(e^{-\gamma_{n} x}-1\right) .
$$

If $\alpha_{n}<x \leq 1$, then

$$
\begin{aligned}
M_{n}(x) & =-\frac{1}{\gamma_{n}}\left(e^{-n}-1\right)+e^{-n} \int_{\alpha_{n}}^{x} e^{\left(s-\alpha_{n}\right) / \delta_{n}} d s \\
& =\frac{1-e^{-n}}{\gamma_{n}}+\delta_{n} e^{-n}\left(e^{\left(x-\alpha_{n}\right) / \delta_{n}}-1\right) .
\end{aligned}
$$

Now we will calculate $\Phi\left(b_{n}\right)=2 \int_{0}^{1} \varphi_{n}^{\prime}(x) M_{n}(x) d x$.

$$
\begin{aligned}
\int_{0}^{\alpha_{n}} \varphi_{n}^{\prime}(x) M_{n}(x) d x & =-\frac{1}{\gamma_{n}} \int_{0}^{\alpha_{n}} e^{\gamma_{n} x}\left(e^{-\gamma_{n} x}-1\right) d x \\
& =-\frac{1}{\gamma_{n}}\left[\alpha_{n}-\int_{0}^{\alpha_{n}} e^{\gamma_{n} x} d x\right] \\
& =-\frac{\alpha_{n}}{\gamma_{n}}+\frac{e^{n}-1}{\gamma_{n}^{2}} .
\end{aligned}
$$

Since $\left(1-\alpha_{n}\right) / \delta_{n}=2+n$,

$$
\begin{aligned}
\int_{\alpha_{n}}^{1} \varphi_{n}^{\prime}(x) M_{n}(x) d x \\
\quad=\int_{\alpha_{n}}^{1} e^{n} e^{-\left(x-\alpha_{n}\right) / \delta_{n}}\left[\frac{1-e^{-n}}{\gamma_{n}}+\delta_{n}\left(e^{-n} e^{\left(x-\alpha_{n}\right) / \delta_{n}}-e^{-n}\right)\right] d x \\
\quad=\frac{e^{n}-1}{\gamma_{n}} \int_{\alpha_{n}}^{1} e^{-\left(x-\alpha_{n}\right) / \delta_{n}} d x+\delta_{n} \int_{\alpha_{n}}^{1} d x-\delta_{n} \int_{\alpha_{n}}^{1} e^{-\left(x-\alpha_{n}\right) / \delta_{n}} d x \\
\quad=-\frac{\left(e^{n}-1\right) \delta_{n}}{\gamma_{n}}\left(e^{-\left(1-\alpha_{n}\right) / \delta_{n}}-1\right)+\delta_{n}\left(1-\alpha_{n}\right)+\delta_{n}^{2}\left(e^{-\left(1-\alpha_{n}\right) / \delta_{n}}-1\right) \\
\quad=\delta_{n} \frac{\left(e^{n}-1\right)}{\gamma_{n}}\left(1-e^{-(2+n)}\right)+\delta_{n}\left(1-\alpha_{n}\right)+\delta_{n}^{2}\left(e^{-(2+n)}-1\right) .
\end{aligned}
$$


Continuing our calculation of $\Phi\left(b_{n}\right)$, we obtain

$$
\begin{aligned}
\frac{1}{2} \Phi\left(b_{n}\right)= & \int_{0}^{1} \varphi_{n}^{\prime}(x) M_{n}(x) d x \\
= & -\frac{\alpha_{n}}{\gamma_{n}}+\frac{e^{n}-1}{\gamma_{n}^{2}}+\delta_{n} \frac{\left(e^{n}-1\right)}{\gamma_{n}}\left(1-e^{-(2+n)}\right) \\
& +\delta_{n}\left(1-\alpha_{n}\right)+\delta_{n}^{2}\left(e^{-(2+n)}-1\right) .
\end{aligned}
$$

As $n \rightarrow \infty, \gamma_{n} \rightarrow \infty, \alpha_{n} \rightarrow 0, \delta_{n} \rightarrow 0$, and

$$
\frac{e^{n}-1}{\gamma_{n}}=\frac{e^{n}-1}{n e^{n}} \rightarrow 0 .
$$

Therefore, $\Phi\left(b_{n}\right) \rightarrow 0$ as $n \rightarrow \infty$.

\section{Proof of Theorem 2 and Theorem 3-}

INFIMUM IN THE CLASS OF PROBABILITY MEASURES

As before, let $\varphi^{\prime}(b, x)=\exp (-2 b[0, x])$ and $\Phi$ be the functional given by (6). We remark that the value of $\Phi\left(x_{0}, b\right)$ will be the same, regardless of whether the interval in the definition of $\varphi^{\prime}$ contains its end points or not. This is because $b$ has at most countably many atoms. In addition, substituting $b\{0\} b(0, x]$ for $b[0, x]$ in the definition of $\varphi^{\prime}$ reveals that if $b$ has an atom at 0 , then the atom has no effect on the value of $\Phi\left(x_{0}, b\right)$, confirming our intuition that any drift at the reflecting boundary point is like having no drift at all. Thus, an explanation of the example given in $\S 2$ is that the function $b_{n}$ is akin to the measure with an atom at 0 of mass $-n$ (which has no effect) and with constant density on $x>0$, the constant equal to $n+1$.

Theorem 2 will follow from the two propositions stated below. Let $b_{1}$ and $b_{2}$ be two arbitrary probability measures on $[0,1]$.

Proposition 1. For each fixed $x_{0}$ and for all $0<t<1$,

$$
\frac{d^{2} \Phi}{d t^{2}}\left(x_{0},(1-t) b_{1}+t b_{2}\right) \geq 0 .
$$

Moreover, if $b_{1}(0, x] \neq b_{2}(0, x]$ for some $x \geq x_{0}$, then for all $0<t<1$,

$$
\frac{d^{2} \Phi}{d t^{2}}\left(x_{0},(1-t) b_{1}+t b_{2}\right)>0 \text {. }
$$

Proposition 2. If $b_{0}$ is the measure given by Theorem 2 and $b$ is any probability measure on $[0,1]$, then

$$
\lim _{t \rightarrow 0^{+}} \frac{d \Phi}{d t}\left(x_{0},(1-t) b_{0}+t b\right) \geq 0 .
$$

Moreover, if $b\left[0, x_{0}\right)>0$, then

$$
\lim _{t \rightarrow 0^{+}} \frac{d \Phi}{d t}\left(x_{0},(1-t) b_{0}+t b\right)>0 .
$$

If $b$ is a probability measure on $[0,1]$ and $b \neq b_{0}$, then $\Phi\left(x_{0}, b_{0}\right)<$ $\Phi\left(x_{0}, b\right)$. Since $b \neq b_{0}, b\left(0, x_{1}\right] \neq b_{0}\left(0, x_{1}\right]$ for some $0<x_{1}<1$. If $x_{1} \geq x_{0}$, then by the second clause of Proposition $1, d^{2} \Phi / d t^{2}>0$ for all $0<t<1$. By Proposition 2, $\lim _{t \rightarrow 0^{+}} d \Phi / d t \geq 0$. Hence, as a function of 
$t, \Phi$ is strictly increasing; in particular, $\Phi\left(x_{0}, b_{0}\right)<\Phi\left(x_{0}, b\right)$. If $x_{1}<x_{0}$, then $b\left(0, x_{1}\right]>0$ since $b_{0}\left(0, x_{1}\right]=0$. By Proposition $1, d^{2} \Phi / d t^{2} \geq 0$ for all $0<t<1$. By the second clause of Proposition 2, $\lim _{t \rightarrow 0^{+}} d \Phi / d t>0$. Again, it follows that $\Phi$ is a strictly increasing function.

Proof of Proposition 1. Let $0 \leq s<y \leq 1$. For any $0 \leq t \leq 1$, by Jensen's inequality

$$
\begin{aligned}
\exp \{ & \left.-2\left((1-t) b_{1}(s, y]+t b_{2}(s, y]\right)\right\} \\
& \leq(1-t) \exp \left\{-2 b_{1}(s, y]\right\}+t \exp \left\{-2 b_{2}(s, y]\right\} .
\end{aligned}
$$

The above inequality is a strict inequality if $b_{1}(s, y] \neq b_{2}(s, y]$ because exp is a strictly convex function. Since

$$
\Phi(c)=2 \int_{x_{0}}^{1} \int_{0}^{y} e^{-2 c(s, y]} d s d y,
$$

by inequality (7),

$$
\Phi\left((1-t) b_{1}+t b_{2}\right) \leq(1-t) \Phi\left(b_{1}\right)+t \Phi\left(b_{2}\right) .
$$

Moreover, if $b_{1}\left(0, x_{1}\right] \neq b_{2}\left(0, x_{1}\right]$ for some $x_{1} \geq x_{0}$, then $b_{1}(s, y] \neq b_{2}(s, y]$ in a small neighborhood around the point $s=0, y=x_{1}$. Hence, in this neighborhood, strict inequality holds in (7), which means strict inequality holds in (8).

Proof of Proposition 2. Let $c(t)=(1-t) b_{0}+t b$, where $b_{0}$ is the measure defined in Theorem 2 and $b$ is an arbitrary measure in $\mathscr{A}$. Let $\varphi^{\prime}(t, x)$ be an abbreviation for $\varphi^{\prime}(c(t), x)$. We will also write $\frac{d \Phi}{d t}(t)$ instead of $\frac{d \Phi}{d t}\left(x_{0}, c(t)\right)$. Because $\varphi^{\prime}(t, x)=\exp \left\{-2\left((1-t) b_{0}[0, x]+t b[0, x]\right)\right\}$,

$$
\frac{\partial \varphi^{\prime}}{\partial t}(t, x)=\varphi^{\prime}(t, x)\left(2 b_{0}[0, x]-2 b[0, x]\right) .
$$

Let $D(x)=b_{0}[0, x]-b[0, x]$. Then

$$
\frac{\partial \varphi^{\prime}}{\partial t}(t, x)=2 \varphi^{\prime}(t, x) D(x) \text {. }
$$

Similarly, $1 / \varphi^{\prime}(t, x)=\exp \left\{2\left((1-t) b_{0}[0, x]+t b[0, x]\right)\right\}$, and

$$
\frac{\partial}{\partial t}\left(\frac{1}{\varphi^{\prime}(t, x)}\right)=-2\left(\frac{1}{\varphi^{\prime}(t, x)}\right) D(x) \text {. }
$$

In Proposition 4 we will prove that differentiating underneath the integral sign is valid when differentiating $\Phi$, the functional which was defined in (6). This fact, plus the last two formulas, yield

$$
\frac{d \Phi}{d t}(t)=4\left\{\int_{x_{0}}^{1} \varphi^{\prime}(t, x) D(x) \int_{0}^{x} \frac{d s}{\varphi^{\prime}(t, s)} d x-\int_{x_{0}}^{1} \varphi^{\prime}(t, x) \int_{0}^{x} \frac{D(s)}{\varphi^{\prime}(t, s)} d s d x\right\} .
$$

Switching the order of integration in the second term, we obtain

$$
\begin{aligned}
\frac{1}{4} \frac{d \Phi}{d t}(t)= & \int_{x_{0}}^{1} \varphi^{\prime}(t, x) D(x) \int_{0}^{x} \frac{d s}{\varphi^{\prime}(t, s)} d x-\int_{x_{0}}^{1} \frac{D(s)}{\varphi^{\prime}(t, s)} \int_{s}^{1} \varphi^{\prime}(t, x) d x d s \\
& -\left(\varphi(t, 1)-\varphi\left(t, x_{0}\right)\right) \int_{0}^{x_{0}} \frac{D(s)}{\varphi^{\prime}(t, s)} d s .
\end{aligned}
$$


Interchanging the roles of $x$ and $s$ in the second integral and letting $t$ go to zero, we obtain

$$
\begin{aligned}
\lim _{t \rightarrow 0^{+}} \frac{1}{4} \frac{d \Phi}{d t}(t)= & \int_{x_{0}}^{1} \varphi^{\prime}(0, x) D(x) \int_{0}^{x} \frac{d s}{\varphi^{\prime}(0, s)} d x \\
& -\int_{x_{0}}^{1} \frac{D(x)}{\varphi^{\prime}(0, x)} \int_{x}^{1} \varphi^{\prime}(0, s) d s d x \\
& -\left(\varphi(0,1)-\varphi\left(0, x_{0}\right)\right) \int_{0}^{x_{0}} \frac{D(s)}{\varphi^{\prime}(0, s)} d s .
\end{aligned}
$$

Taking the limit underneath the integral is permitted by Lebesgue's Dominated Convergence Theorem because each of the functions $D(x), \varphi^{\prime}(t, x), 1 / \varphi^{\prime}(t, x)$, is bounded on $0 \leq x \leq 1$, uniformly in $t$. For ease of notation, let $\varphi^{\prime}(x)=$ $\varphi^{\prime}\left(b_{0}, x\right)$ and $M(x)=M\left(b_{0}, x\right)$. Let

$$
\begin{aligned}
& I_{1}=\int_{x_{0}}^{1} \varphi^{\prime}(x) D(x) \int_{0}^{x} \frac{d s}{\varphi^{\prime}(s)} d x=\int_{x_{0}}^{1} \varphi^{\prime}(x) D(x) M(x) d x, \\
& I_{2}=-\int_{x_{0}}^{1} \frac{D(x)}{\varphi^{\prime}(x)} \int_{x}^{1} \varphi^{\prime}(s) d s d x=-\int_{x_{0}}^{1} \frac{D(x)}{\varphi^{\prime}(x)}[\varphi(1)-\varphi(x)] d x, \\
& I_{3}=-\left(\varphi(1)-\varphi\left(x_{0}\right)\right) \int_{0}^{x_{0}} \frac{D(s)}{\varphi^{\prime}(s)} d s .
\end{aligned}
$$

Then $\lim _{t \rightarrow 0+} d \Phi / d t=4\left(I_{1}+I_{2}+I_{3}\right)$. We will prove Proposition 2 by showing that $I_{3} \geq 0$ and $I_{1}+I_{2} \geq 0$. If, in addition, $b$ gives positive weight to $\left[0, x_{0}\right)$, then $I_{3}>0$.

Lemma 1. Given any probability measure $b$ on $[0,1], I_{3} \geq 0$. If, in addition, $b\left[0, x_{0}\right)>0$, then $I_{3}>0$.

Proof. As before, let $D(x)=b_{0}[0, x]-b[0, x]$. On $0<x<x_{0}, D(x)=$ $-b[0, x]$ because $b_{0}\left[0, x_{0}\right)=0$. Hence, $I_{3}$ can be rewritten as

$$
I_{3}=\left(\varphi(1)-\varphi\left(x_{0}\right)\right) \int_{0}^{x_{0}} \frac{b[0, s]}{\varphi^{\prime}(s)} d s .
$$

Because $\varphi^{\prime}>0$ and $\varphi(1)-\varphi\left(x_{0}\right)>0, I_{3}$ must be nonnegative. In addition, if $b\left[0, x_{0}\right)>0$, then $I_{3}>0$.

Let $f$ be the function

$$
\begin{aligned}
f(x) & =\varphi^{\prime}(x) M(x)-\frac{[\varphi(1)-\varphi(x)]}{\varphi^{\prime}(x)} \\
& =e^{-2 b_{0}[0, x]} \int_{0}^{x} e^{2 b_{0}[0, s]} d s-e^{2 b_{0}[0, x]} \int_{x}^{1} e^{-2 b_{0}[0, s]} d s .
\end{aligned}
$$

Then $I_{1}+I_{2}=\int_{x_{0}}^{1} D(x) f(x) d x$, and a routine calculation shows that $\left(G(x, x) u^{\prime}(x)\right)^{\prime}=4 f$, where $G(x, y)$ is the Green's function for our process killed when it hits 1 , and $u(x)=E_{x} T_{1}$. Now we will calculate $f$ explicitly and show that for any probability measure $b$ on $[0,1], I_{1}+I_{2}$ is nonnegative. There are three cases, depending on the value of $x_{0}$. 
Lemma 2. $I_{1}+I_{2} \geq 0$. Case $0 \leq x_{0} \leq 1 / 4$.

Proof. In this case, $b_{0}$ is the function which is 2 on the interval [1/4,3/4] and is 0 otherwise. A routine calculation shows that

$$
\varphi^{\prime}(x)=e^{-2 b_{0}[0, x]}= \begin{cases}1 & \text { if } 0 \leq x<\frac{1}{4} \\ e^{-4 x+1} & \text { if } \frac{1}{4} \leq x \leq \frac{3}{4} \\ e^{-2} & \text { if } \frac{3}{4}<x \leq 1 .\end{cases}
$$

As before, let $M(x)=\int_{0}^{x} e^{2 b_{0}[0, x]} d z$. Then

$$
M(x)= \begin{cases}x & \text { if } 0 \leq x<\frac{1}{4} \\ \frac{1}{4} e^{4 x-1} & \text { if } \frac{1}{4} \leq x \leq \frac{3}{4}, \\ e^{2}\left(x-\frac{1}{2}\right) & \text { if } \frac{3}{4}<x \leq 1 .\end{cases}
$$

We also need to calculate $\varphi(1)-\varphi(x)=\int_{x}^{1} \varphi^{\prime}(z) d z$.

$$
\varphi(1)-\varphi(x)= \begin{cases}e^{-2}(1-x) & \text { if } \frac{3}{4}<x \leq 1 \\ \frac{1}{4} e^{-4 x+1} & \text { if } \frac{1}{4} \leq x \leq \frac{3}{4} \\ \frac{1}{2}-x & \text { if } 0 \leq x<\frac{1}{4}\end{cases}
$$

We calculate $f$ using (10)-(12). On $0 \leq x<1 / 4$,

$$
\begin{aligned}
f(x) & =\varphi^{\prime}(x) M(x)-\frac{[\varphi(1)-\varphi(x)]}{\varphi^{\prime}(x)} \\
& =x-\left(\frac{1}{2}-x\right)=2 x-\frac{1}{2}<0 .
\end{aligned}
$$

On $1 / 4 \leq x \leq 3 / 4, f(x)=\frac{1}{4}-\frac{1}{4}=0$. To reiterate, $f$ is 0 on [1/4, 3/4], i.e., $G(x, x) u^{\prime}(x)$ is constant when $b_{0}(x)$ is strictly positive. This is a crucial property of the rather ordinary-looking step function $b_{0}(x)$. On $3 / 4<x<1$,

$$
f(x)=x-\frac{1}{2}-(1-x)=2 x-\frac{3}{2}>0 .
$$

We have the identities: $D(x)=b_{0}[0, x]-b[0, x]=-b[0, x]$ on $x<1 / 4$ and $D(x)=1-b[0, x]$ on $x>3 / 4$. Because $b$ is a probability measure on $[0,1], 1-b[0, x] \geq 0$. Hence,

$$
\begin{aligned}
I_{1}+I_{2}= & \int_{x_{0}}^{1} D(x) f(x) d x \\
= & -\int_{x_{0}}^{1 / 4} b[0, x]\left(2 x-\frac{1}{2}\right) d x \\
& +\int_{3 / 4}^{1}(1-b[0, x])\left(2 x-\frac{3}{2}\right) d x \geq 0 .
\end{aligned}
$$

Lemma 3. $I_{1}+I_{2} \geq 0$. Case $1 / 4<x_{0}<1 /\left(e^{-2}+1\right)$.

Proof. In this case, $b_{0}$ is the sum of a delta function and a step function. Let $h=\left(x_{1}-x_{0}\right) / 2(1-w)$. Then equation (5) can be rewritten as $h=x_{0} e^{-2 w}=$ $1-x_{1}$, and the density given by (4) can be rewritten as $b_{0}(x)=1 /(2 h)$ on $x_{0}<x<x_{1}$. Because $w+\left\{\left(x_{1}-x_{0}\right) /(2 h)\right\}=1, \varphi^{\prime}$ equals

$$
\varphi^{\prime}(x)=e^{-2 b_{0}[0, x]}= \begin{cases}1 & \text { if } 0 \leq x<x_{0}, \\ e^{-2 w} e^{-\left(x-x_{0}\right) / h} & \text { if } x_{0} \leq x \leq x_{1}, \\ e^{-2} & \text { if } x>x_{1} .\end{cases}
$$


A routine calculation shows that $M(x)=\int_{0}^{x} e^{2 b_{0}[0, z]} d z$ is given by

$$
M(x)= \begin{cases}x & \text { if } 0 \leq x<x_{0} \\ h e^{2 w} e^{\left(x-x_{0}\right) / h} & \text { if } x_{0} \leq x \leq x_{1} \\ e^{2}\left(h+x-x_{1}\right) & \text { if } x>x_{1}\end{cases}
$$

Lastly, we will calculate $\varphi(1)-\varphi(x)=\int_{x}^{1} \varphi^{\prime}(z) d z$. (It will not be necessary to compute $\varphi(1)-\varphi(x)$ for $x<x_{0}$.)

$$
\varphi(1)-\varphi(x)= \begin{cases}e^{-2}(1-x) & \text { if } x>x_{1}, \\ h e^{-2 w} e^{-\left(x-x_{0}\right) / h} & \text { if } x_{0} \leq x \leq x_{1} .\end{cases}
$$

We proceed to calculate $f$, this time using (13)-(15). On $x_{0} \leq x \leq x_{1}$, $f(x)=h-h=0$. On $x>x_{1}$, since $h=1-x_{1}$,

$$
\begin{aligned}
f(x) & =\varphi^{\prime}(x) M(x)-\frac{[\varphi(1)-\varphi(x)]}{\varphi^{\prime}(x)} \\
& =h+x-x_{1}-(1-x)=2 x-x_{1}+h-1 \\
& \geq x_{1}+h-1=0 .
\end{aligned}
$$

Consequently, using the identity $D(x)=1-b[0, x]$ in the region $x \geq x_{1}$, it follows that

$$
\begin{aligned}
I_{1}+I_{2} & =\int_{x_{0}}^{1} D(x) f(x) d x \\
& =\int_{x_{1}}^{1}(1-b[0, x])\left(2 x-x_{1}+h-1\right) d x \geq 0 .
\end{aligned}
$$

Lemma 4. $I_{1}+I_{2} \geq 0$. Case $x_{0} \geq 1 /\left(e^{-2}+1\right)$.

Proof. Now the minimizing measure is an atom at $x_{0}$, so the calculations are rather easy. We restrict our attention to the interval $x \geq x_{0}$. On this interval, $\varphi^{\prime}(x)=e^{-2}, M(x)=e^{2} x+x_{0}\left(1-e^{2}\right)$, and $\varphi(1)-\varphi(x)=e^{-2}(1-x)$. On $x \geq x_{0} \geq 1 /\left(e^{-2}+1\right)$,

$$
\begin{aligned}
f(x) & =\varphi^{\prime}(x) M(x)-\frac{[\varphi(1)-\varphi(x)]}{\varphi^{\prime}(x)} \\
& =x+x_{0}\left(e^{-2}-1\right)-(1-x) \\
& =2 x+x_{0}\left(e^{-2}-1\right)-1 \\
& \geq x_{0}\left(e^{-2}+1\right)-1 \geq 0 .
\end{aligned}
$$

Thus, it follows that

$$
\begin{aligned}
I_{1}+I_{2} & =\int_{x_{0}}^{1} D(x) f(x) d x \\
& =\int_{x_{0}}^{1}(1-b[0, x])\left(2 x+x_{0}\left(e^{-2}-1\right)-1\right) d x \geq 0 .
\end{aligned}
$$

We will now evaluate $\Phi\left(x_{0}, b_{0}\right)=\inf _{b \in \mathscr{A}_{m}} \Phi\left(x_{0}, b\right)$. 
Proposition 3. The value of $\Phi\left(x_{0}, b_{0}\right)$ is as given by Theorem 2 .

Proof. Again, there are three cases.

Case $0 \leq x_{0} \leq 1 / 4$. In general, $\Phi\left(x_{0}, b\right)=2 \int_{x_{0}}^{1} \varphi^{\prime}(b, x) M(b, x) d x$. Using equations (10) and (11), it follows that

$$
\begin{aligned}
\Phi\left(x_{0}, b_{0}\right) & =2\left\{\int_{x_{0}}^{1 / 4} x d x+\frac{1}{4} \int_{1 / 4}^{3 / 4} d x+\int_{3 / 4}^{1}\left(x-\frac{1}{2}\right) d x\right\} \\
& =2\left\{\frac{1}{2}\left(\frac{1}{16}-x_{0}^{2}\right)+\frac{1}{8}+\frac{3}{32}\right\} \\
& =2\left\{\frac{1}{4}-\frac{1}{2} x_{0}^{2}\right\}=\frac{1}{2}-x_{0}^{2} .
\end{aligned}
$$

Case $1 / 4<x_{0}<1 /\left(e^{-2}+1\right)$. Since $h=\left(x_{1}-x_{0}\right) / 2(1-w)=1-x_{1}$, using equations (13) and (14), we obtain

$$
\begin{aligned}
\Phi\left(x_{0}, b_{0}\right) & =2\left\{h\left(x_{1}-x_{0}\right)+\int_{x_{1}}^{1} h+x-x_{1} d x\right\} \\
& =2\left\{h\left(x_{1}-x_{0}\right)+\frac{1}{2}\left[\left(h+1-x_{1}\right)^{2}-h^{2}\right]\right\} \\
& =2\left\{\left(1-x_{1}\right)\left(x_{1}-x_{0}\right)+\frac{3}{2}\left(1-x_{1}\right)^{2}\right\} \\
& =2\left\{\left(1-x_{1}\right)\left[\frac{3}{2}-x_{0}-\frac{1}{2} x_{1}\right]\right\} \\
& =\left(1-x_{1}\right)\left(3-2 x_{0}-x_{1}\right) .
\end{aligned}
$$

Case $x_{0} \geq 1 /\left(e^{-2}+1\right)$.

$$
\begin{aligned}
\Phi\left(x_{0}, b_{0}\right) & =2\left\{\frac{1}{2}\left(1-x_{0}^{2}\right)+e^{-2} x_{0}\left(1-e^{2}\right)\left(1-x_{0}\right)\right\} \\
& =2\left\{\left(1-x_{0}\right)\left[\frac{1}{2}+\frac{1}{2} x_{0}+x_{0}\left(e^{-2}-1\right)\right]\right\} \\
& =2\left\{\left(1-x_{0}\right)\left[\frac{1}{2}+x_{0}\left(e^{-2}-\frac{1}{2}\right)\right]\right\} \\
& =\left(1-x_{0}\right)\left(1+x_{0}\left(2 e^{-2}-1\right)\right) .
\end{aligned}
$$

This ends the proof of Theorem 2.

Proof of Theorem 3. Let $\delta_{x}$ be the unit mass concentrated at $x$. Let $b$ be any probability measure on $[0,1]$ different from $\delta_{x}$. Proceeding as in the proof of Theorem 2, let $\varphi^{\prime}(t, z)=\exp \left\{-2\left((1-t) \delta_{x}[0, z]+t b[0, z]\right)\right\}$. For convenience, we will write $\Psi(x, t)$ instead of $\Psi\left(x,(1-t) \delta_{x}+t b\right)$. Then

$$
\Psi(x, t)=P_{x}\left(T_{1}<T_{0}\right)=\frac{\int_{0}^{x} \varphi^{\prime}(t, z) d z}{\int_{0}^{1} \varphi^{\prime}(t, z) d z} .
$$

As mentioned earlier, $\partial \varphi^{\prime} / \partial t=2 D \varphi^{\prime}$, where $D(z)=\delta_{x}[0, z]-b[0, z]$. Differentiating underneath the integral (see Proposition 4 for justification), we 
obtain

(16)

$$
\begin{aligned}
\frac{d \Psi}{d t}(x, t)=2 & \left\{\int_{0}^{1} \varphi^{\prime}(t, z) d z \int_{0}^{x} \varphi^{\prime}(t, z) D(z) d z\right. \\
& \left.-\int_{0}^{x} \varphi^{\prime}(t, z) d z \int_{0}^{1} \varphi^{\prime}(t, z) D(z) d z\right\} /\left(\int_{0}^{1} \varphi^{\prime}(t, z) d z\right)^{2} .
\end{aligned}
$$

Now we will show that the numerator of $d \Psi / d t$ is strictly negative. Looking at the terms in the numerator inside the braces, if $\int_{0}^{1} \varphi^{\prime}(t, z) d z$ is rewritten as the sum $\int_{0}^{x} \varphi^{\prime}(t, z) d z+\int_{x}^{1} \varphi^{\prime}(t, z) d z$, then cancellation occurs and

$$
\begin{aligned}
\int_{0}^{1} \varphi^{\prime}(t, z) d z \int_{0}^{x} \varphi^{\prime}(t, z) D(z) d z-\int_{0}^{x} \varphi^{\prime}(t, z) d z \int_{0}^{1} \varphi^{\prime}(t, z) D(z) d z \\
\quad=\int_{x}^{1} \varphi^{\prime}(t, z) d z \int_{0}^{x} \varphi^{\prime}(t, z) D(z) d z-\int_{0}^{x} \varphi^{\prime}(t, z) d z \int_{x}^{1} \varphi^{\prime}(t, z) D(z) d z .
\end{aligned}
$$

We look at each integral separately. Since $\varphi^{\prime}>0$ and $D(z)=-b[0, z] \leq 0$ on $0 \leq z<x$, it follows that

$$
\begin{aligned}
\int_{x}^{1} \varphi^{\prime}(t, z) d z \int_{0}^{x} \varphi^{\prime}(t, z) D(z) d z \\
=\int_{x}^{1} \varphi^{\prime}(t, z) d z \int_{0}^{x} \varphi^{\prime}(t, z)(-b[0, z]) d z \leq 0 .
\end{aligned}
$$

Similarly, because $D(z)=1-b[0, z] \geq 0$ on $z \geq x$, it follows that

$$
\begin{aligned}
-\int_{0}^{x} \varphi^{\prime}(t, z) d z \int_{x}^{1} \varphi^{\prime}(t, z) D(z) d z \\
\quad=-\int_{0}^{x} \varphi^{\prime}(t, z) d z \int_{x}^{1} \varphi^{\prime}(t, z)(1-b[0, z]) d z \leq 0 .
\end{aligned}
$$

Furthermore, at least one of the last two equations, (17) and (18), must be strictly negative since by assumption $b$ is different from $\delta_{x}$. Thus, the numerator of $d \Psi / d t$ is strictly negative. The denominator of $d \Psi / d t$ is strictly positive. Hence, for all $t, 0<t<1, d \Psi / d t<0$. On other words, as a function of $t, \Psi$ is a strictly decreasing function. Consequently, $\Psi\left(x, \delta_{x}\right)>\Psi(x, b)$ for any $b \neq \delta_{z}$.

Now we will justify differentiating underneath the integral sign.

Proposition 4. The derivatives, $d \Phi / d t$ and $d \Psi / d t$, are as given by (9) and (16), respectively.

We begin with a lemma.

Lemma 5. Let $a$ and $b$ be constants, $a<b$; and let $h(t)=\int_{a}^{b} g(t, x) d x$. Assume that for each fixed $x, \partial g / \partial t$ is continuous with respect to $t$. Assume also that $|\partial g / \partial t| \leq M$ for some constant $M$. Then

$$
\frac{d h}{d t}(t)=\int_{a}^{b} \frac{\partial g}{\partial t}(t, x) d x .
$$


Proof. By the Fundamental Theorem of Calculus,

$$
h(t+\Delta t)-h(t)=\int_{a}^{b} \int_{t}^{t+\Delta t} \frac{\partial g}{\partial t}(u, x) d u d x .
$$

By assumption, $\partial g / \partial t$ is continuous with respect to $t$. By the Mean Value Theorem, there exists some $v, t \leq v \leq t+\Delta t$, such that

$$
\frac{1}{\Delta t} \int_{t}^{t+\Delta t} \frac{\partial g}{\partial t}(u, x) d u=\frac{\partial g}{\partial t}(v, x) \text {. }
$$

As $\Delta t \rightarrow 0, v \rightarrow t$. Since $\partial g / \partial t$ is continuous with respect to $t$,

$$
\lim _{\Delta t \rightarrow 0} \frac{1}{\Delta t} \int_{t}^{t+\Delta t} \frac{\partial g}{\partial t}(u, x) d u=\frac{\partial g}{\partial t}(t, x) .
$$

Because $|\partial g / \partial t| \leq M$,

$$
\left|\frac{1}{\Delta t} \int_{t}^{t+\Delta t} \frac{\partial g}{\partial t}(u, x) d u\right|=\left|\frac{\partial g}{\partial t}(v, x)\right| \leq M .
$$

By Lebesgue's Dominated Convergence Theorem,

$$
\begin{aligned}
\frac{d h}{d t}(t) & =\lim _{\Delta t \rightarrow 0} \frac{1}{\Delta t} \int_{a}^{b} \int_{t}^{t+\Delta t} \frac{\partial g}{\partial t}(u, x) d u d x \\
& =\int_{a}^{b} \frac{\partial g}{\partial t}(t, x) d x .
\end{aligned}
$$

Clearly, $1 / \varphi^{\prime}(t, s)=\exp \left\{2\left((1-t) b_{1}[0, s]+t b_{2}[0, s]\right)\right\}$ is a differentiable function of $t$ and so is

$$
\frac{\partial}{\partial t}\left(\frac{1}{\varphi^{\prime}(t, s)}\right)=-2\left(\frac{1}{\varphi^{\prime}(t, s)}\right) D(s) .
$$

Furthermore, $|D(s)| \leq 1$ and $1 \leq 1 / \varphi^{\prime}(t, s) \leq e^{2}$. By Lemma 5 , we can differentiate the function $M(t, x)=\int_{0}^{x}\left(1 / \varphi^{\prime}(t, s)\right) d s$ underneath the integral sign and conclude that

$$
\frac{\partial M}{\partial t}(t, x)=-2 \int_{0}^{x} \frac{D(s)}{\varphi^{\prime}(t, s)} d s .
$$

We will now focus on $\Phi$, using arguments similar to that above. Let

$$
f(t, x)=\varphi^{\prime}(t, x) M(t, x)=\varphi^{\prime}(t, x) \int_{0}^{x} \frac{d s}{\varphi^{\prime}(t, s)} .
$$

Then, $\Phi\left(x_{0}, t\right)=2 \int_{x_{0}}^{1} f(t, x) d x$. We start with the observation that

$$
\frac{\partial f}{\partial t}=2\left\{\varphi^{\prime}(t, x) D(x) \int_{0}^{x} \frac{d s}{\varphi^{\prime}(t, s)}-\varphi^{\prime}(t, x) \int_{0}^{x} \frac{D(s)}{\varphi^{\prime}(t, s)} d s\right\} .
$$

The function $\partial f / \partial t$ is continuous with respect to $t$. It is also bounded because, as stated earlier, $|D(x)| \leq 1$ and $e^{-2} \leq \varphi^{\prime}(t, x) \leq 1$. Again, by Lemma 5 , we can differentiate underneath the integral sign and conclude that $d \Phi / d t=$ $2 \int_{x_{0}}^{1}(\partial f / \partial t) d x$. Analogous reasoning shows that $d \Psi / d t$ is given by (16). 


\section{Proof of Theorem 4-Construction of the diffusion Process}

In order to take into account reflection at 0 , we will first define what it means for a measure $\bar{b}$ to be the odd reflection of a given measure $b$. Next, we will show that there exists a solution $X_{t}$ to the SDE $d X_{t}=d B_{t}+\bar{b}\left(X_{t}\right) d t$. Lastly, we will show that the expected time for $X_{t}$ to hit 1 , starting from $X_{0}=x$, equals $u(x)$ where $u$ is the function defined by (2).

Given a probability measure $b$ on $[0,1]$, extend it to a probability measure on the positive real line $[0, \infty)$ by the rule $b(A)=b(A \cap[0,1])$. As noted earlier, if $b$ has an atom at $\{0\}$, the atom does not affect the value of $\Phi\left(x_{0}, b\right)$. Therefore, we shall assume $b$ does not have an atom at 0 . (This will simplify a few arguments.)

Let $\bar{b}$ be the signed measure on $\mathbb{R}$ defined by: if $x \geq 0$, then $\bar{b}[0, x]=$ $b[0, x]$. If $x<0$, then $\bar{b}(x, 0)=-b(0,-x)$. Call $\bar{b}$ the odd reflection of $b$ about zero. Recalling the definition of $\varphi^{\prime}$,

$$
\varphi^{\prime}(\bar{b}, x)= \begin{cases}e^{-2 \bar{b}[0, x]}=e^{-2 b[0, x]} & \text { if } x \geq 0, \\ e^{2 \bar{b}(x, 0)}=e^{-2 b(0,-x)} & \text { if } x<0 .\end{cases}
$$

For ease of notation, let $\varphi^{\prime}(y)$ be shorthand for $\varphi^{\prime}(\bar{b}, y)$. Let $\varphi(x)=$ $\int_{0}^{x} \varphi^{\prime}(y) d y$. Because $\varphi^{\prime}>0, \varphi$ is a strictly increasing function and $\varphi^{-1}$ exists. Let $I=\varphi^{-1}$, and let $g(x)=\varphi^{\prime}(I(x))=\varphi^{\prime}\left(\varphi^{-1}(x)\right)$.

Lemma 6. There exists a solution to $d Y_{t}=g\left(Y_{t}\right) d B_{t}$.

Proof. We will construct $Y_{t}$ by time-changing Brownian motion. Given a Brownian motion, $W_{t}$, let

$$
\Gamma(t)=\int_{0}^{t} \frac{d s}{g^{2}\left(W_{s}\right)} .
$$

Let $\gamma=\Gamma^{-1}$ and let $Y_{t}=W_{\gamma(t)}$. Since $1 \leq 1 / g^{2} \leq e^{4}, \gamma$ is absolutely continuous and $\gamma^{\prime}$ exists almost surely. Differentiating the identity $\Gamma(\gamma(t))=t$ yields: $\gamma^{\prime}(t)=1 / \Gamma^{\prime}(\gamma(t))=g^{2}\left(W_{\gamma(t)}\right)$. Thus,

$$
\langle Y\rangle_{t}=\gamma(t)=\int_{0}^{t} \gamma^{\prime}(s) d s=\int_{0}^{t} g^{2}\left(Y_{s}\right) d s .
$$

This time change is valid for all time because $t \leq \Gamma(t) \leq e^{4} t$ for all $t \geq 0$. Hence, $Y_{t}$ is a martingale, being a time change of Brownian motion. Let $B_{t}-$ $B_{0}=\int_{0}^{t} g^{-2}\left(Y_{s}\right) d Y_{s}$. Then $B_{t}$ is a Brownian motion because it is a continuous martingale and $\langle B\rangle_{t}=t$. Thus, $d Y_{t}=g\left(Y_{t}\right) d B_{t}$ as desired.

Let $X_{t}=I\left(Y_{t}\right)=\varphi^{-1}\left(Y_{t}\right)$. Now we will show that $X_{t}$ is a solution to the SDE $d X_{t}=d B_{t}+\bar{b}\left(X_{t}\right) d t$. Clearly, $\varphi\left(X_{t}\right)=Y_{t}$ is a martingale. It remains to show that $\langle X\rangle_{t}=t$. We will begin with a lemma. The function $I(x)$ is strictly positive for $x>0$, equals 0 when $x=0$ and is strictly negative for $x<0$. Let $I^{+}(x)$ be the function which equals $I(x)$ if $x \leq 0$ and is 0 otherwise. Let $I^{-}(x)$ be the function which equals $-I(x)$ if $x \leq 0$ and is 0 otherwise.

Lemma 7. $\mathrm{I}^{+}$is convex and $\mathrm{I}^{-}$is convex.

Proof. The function $\varphi^{\prime}(x)$ was defined so that it is even about $x=0$ a.s. Consequently, $\varphi(x)$ is an odd function about $x=0$ and so is its inverse, 
$I(x)=\varphi^{-1}(x)$. Thus, it suffices to show that $I^{+}$is convex, because then by symmetry, $I^{-}$is also convex.

By Proposition 6, left differentiating the identity $\varphi(I(x))=x$ yields: $D_{-} I(x)=1 / D_{-} \varphi(I(x))$. By Proposition $5, D_{-} \varphi$ is the left-continuous version of $\varphi^{\prime}$, where $\varphi^{\prime}$ is the function defined by (19). Consequently, on $x>0$, $D_{-} I(x)=e^{2 \bar{b}[0, I(x))}$, which is a positive, nondecreasing function. Thus $I^{+}$is convex.

Lemma 8. $\langle X\rangle_{t}=t$.

Proof. By Lemma 7, $I=I^{+}-I^{-}$is the difference of two convex functions. We can thus apply Trotter and Meyer's extension of Itô's formula to $I\left(Y_{t}\right)$. (See, for instance, Rogers and Williams [4, Theorem IV.45.1, p. 103].) Let $g_{l}(x)=D_{-} \varphi(I(x))$. Then $D_{-} I(x)=1 / D_{-} \varphi(I(x))=1 / g_{l}(x)$. Consequently,

$$
\begin{aligned}
X_{t}-X_{0} & =I\left(Y_{t}\right)-I\left(Y_{0}\right)=\int_{0}^{t} D_{-} I\left(Y_{s}\right) d Y_{s}+\frac{1}{2} \int_{-\infty}^{\infty} l_{t}^{y} m(d y) \\
& =\int_{0}^{t} \frac{d Y_{s}}{g_{l}\left(Y_{s}\right)}+\frac{1}{2} \int_{-\infty}^{\infty} l_{t}^{y} m(d y)
\end{aligned}
$$

where $l_{t}^{y}$ is the local time of $Y$ at $y$ and $I^{\prime \prime}=m$ (as a measure). Rewriting the last equation using the identity $d Y_{t}=g\left(Y_{t}\right) d B_{t}$ and using the fact that the functions $g(x)$ and $g_{l}(x)$ differ on at most countably many points gives

$$
X_{t}-X_{0}=\int_{0}^{t} d B_{s}+\frac{1}{2} \int_{-\infty}^{\infty} l_{t}^{y} m(d y)
$$

As a function of $t, \int_{-\infty}^{\infty} l_{t}^{y} m(d y)$ is continuous and nondecreasing and hence is of bounded variation. Therefore, $\langle X\rangle_{t}=t$.

Our next step is to check that $u(x)=E_{x} T_{1}$, where $u$ is the function defined by (2) and $\varphi^{\prime}$ is the function defined by (19).

Lemma 9. The process $u\left(X_{t}\right)+t$ is a martingale.

Proof. By definition, $u\left(X_{t}\right)-u\left(X_{0}\right)=u\left(I\left(Y_{t}\right)\right)-u\left(I\left(Y_{0}\right)\right)$. Let $f(x)=u(I(x))$. If $b$ has atoms, then $I^{\prime}$ is discontinuous. However, $f^{\prime}$ still exists and is continuous. By Proposition 5, $D_{-} u(x)$ is the left continuous version of the integrand, $-2 \varphi^{\prime}(x) \int_{0}^{x} d y / \varphi^{\prime}(y)$. By Proposition 6,

$$
\begin{aligned}
& D_{-} f(x)=D_{-} u(I(x)) D_{-} I(x)=-2 \int_{0}^{I(x)} \frac{d s}{\varphi^{\prime}(s)}, \\
& D_{+} f(x)=D_{+} u(I(x)) D_{+} I(x)=-2 \int_{0}^{I(x)} \frac{d s}{\varphi^{\prime}(s)} .
\end{aligned}
$$

In addition, $f^{\prime \prime}$ exists a.e. and is given by

$$
f^{\prime \prime}(x)=\frac{-2 I^{\prime}(x)}{\varphi^{\prime}(I(x))}=\frac{-2}{g^{2}(x)} .
$$

There is an extended version of Itô's formula which applies to functions $f$ such that $f^{\prime}$ is continuous and $f^{\prime \prime}$ is a function in the sense of Schwartz distribution. (See, for example, Rogers and Williams [4, Lemma IV.45.9, p. 
105].) Applying this extended Itô's formula to $f(x)=u(I(x))$ and using the equality $d\langle Y\rangle_{s}=g^{2}\left(Y_{s}\right) d s$, it follows that

$$
\begin{aligned}
u\left(X_{t}\right)-u\left(X_{0}\right) & =f\left(Y_{t}\right)-f\left(Y_{0}\right)=\int_{0}^{t} f^{\prime}\left(Y_{s}\right) d Y_{s}+\frac{1}{2} \int_{0}^{t} f^{\prime \prime}\left(Y_{s}\right) d\langle Y\rangle_{s} \\
& =\int_{0}^{t} f^{\prime}\left(Y_{s}\right) d Y_{s}-\int_{0}^{t} \frac{g^{2}\left(Y_{s}\right)}{g^{2}\left(Y_{s}\right)} d s=\int_{0}^{t} f^{\prime}\left(Y_{s}\right) d Y_{s}-t .
\end{aligned}
$$

The last step is to show

Lemma 10. The function $u(x)$ equals $E_{x} T_{1}$.

Proof. Clearly, $u(1)=0$. Moreover, $u(-1)=0$ because $\varphi^{\prime}(y)$ is even about $y=0$, whereas $M(y)=\int_{0}^{y} d s / \varphi^{\prime}(s)$ is odd about $y=0$. Hence, $\varphi^{\prime}(y) M(y)$ is odd about $y=0$ and $u(-1)=\int_{-1}^{1} \varphi^{\prime}(y) M(y) d y=0$. Let $T_{1}$ be the first time $X_{t}$ exits the interval $(-1,1)$. Then, by the Optional Stopping Theorem, $u(x)=E_{x}\left(u\left(T_{1}\right)+T_{1}\right)=0+E_{x} T_{1}=E_{x} T_{1}$.

For the sake of completeness, we now present two propositions concerning one-sided derivatives. Let $h$ be a real-valued function with finite left and right limits everywhere. Let $h_{l}(x)=\lim _{\Delta x \rightarrow 0^{+}} h(x-\Delta x)$ be the left continuous version of $h$, and let $h_{r}(x)=\lim _{\Delta x \rightarrow 0^{+}} h(x+\Delta x)$ be the right continuous version of $h$. Let $H(x)=\int_{0}^{x} h(y) d y$. Then

Proposition 5. The left-hand derivative of $H, D_{-} H$, equals $h_{l}$ and the righthand derivative of $H, D_{+} H$, equals $h_{r}$.

The proof of Proposition 5 is routine and we omit it here.

Proposition 6. Suppose $f(x)=g(h(x))$. Assume $h$ is a continuous, strictly increasing function. Assume also that both, $g$ and $h$, have left-hand derivatives everywhere. Then $D_{-} f(x)=D_{-} g(h(x)) D_{-} h(x)$.

Proof. Let $y=h(x)$, and let $\Delta k=h(x)-h(x-\Delta x)=y-h(x-\Delta x)$. Because $h$ is a strictly increasing function, $\Delta k>0$. Hence,

$$
\begin{aligned}
\frac{f(x-\Delta x)-f(x)}{-\Delta x} & =\frac{g(y-\Delta k)-g(y)}{-\Delta x}\left(\frac{-\Delta k}{-\Delta k}\right) \\
& =\left(\frac{g(y-\Delta k)-g(y)}{-\Delta k}\right)\left(\frac{h(x-\Delta x)-h(x)}{-\Delta x}\right) .
\end{aligned}
$$

Since $h$ is continuous, $\Delta k \rightarrow 0$ as $\Delta x \rightarrow 0^{+}$. Taking the limit as $\Delta x \rightarrow 0^{+}$of the above equation, it follows that $D_{-} f(x)=D_{-} g(h(x)) D_{-} h(x)$.

\section{ACKNOWLEDGMENT}

The research described here is a part of the author's Ph.D. dissertation. The author would like to thank her advisor, Rick Durrett, for his invaluable guidance and his infinite patience. She would also like to thank five other people: Itai Benjamini, who suggested this problem; Brian Hall, for our many discussions on one-dimensional diffusion; John H. Hubbard, for showing her how to improve the proof of Theorem 2; Robin Pemantle, who corrected her early calculations and made many insightful comments; and Lars Wahlbin, who showed her a proof of Theorem 1 completely different from the one presented here. 


\section{REFERENCES}

1. H. J. Engelbert and W. Schmidt, On one-dimensional stochastic differential equations with generalized drift, Stochastic Differential Systems, Lecture Notes in Control and Information Sciences, vol. 69, Springer-Verlag, New York, 1984, pp. 143-155.

2. J. M. Harrison and L. A. Shepp, On skew Brownian motion, Ann. Probab. 9 (1981), 309-313.

3. R. Lyons, R. Pemantle, and Y. Peres, Ergodic theory on Galton-Watson trees I: Speed of random walk and dimension of harmonic measure, preprint, 1993.

4. L. C. G. Rogers and David Williams, Diffusions, Markov processes and martingales, Vol. 2, Wiley, New York, 1987.

Department of Mathematics, 0112 , University of California at San Diego, La Jolla, CALIFORNIA 92093-0112 